\title{
Territorio y lugar: la espacialidad en debate en la Economía Social y Solidaria. Aproximaciones conceptuales a partir de experiencias rurales concretas
}

- Emanuel Alberto Jurado

Grupo de Estudios Geografías Emergentes, Instituto de Geografía, Facultad de Filosofía y Letras, UBA.

Recibido: 4 de noviembre de 2019. Aceptado: 2 de abril de 2020.

\begin{abstract}
Resumen
En las últimas décadas, asistimos al denominado giro espacial en las ciencias sociales. Se trata de un proceso en marcha donde la espacialidad de los fenómenos sociales adquiere gran relevancia para diferentes enfoques disciplinares. Sin embargo, como investigadores/as nos enfrentamos permanentemente al desafío de la elección de conceptos geográficos que permitan dar cuenta de nuestros objetos-sujetos de análisis. El presente texto busca compartir este dilema teórico, haciendo foco en los conceptos de territorio y lugar como posibles herramientas analíticas para el estudio de procesos productivos asociativos enmarcados en la Economía Social y Solidaria. Para ello, desarrollamos algunos aspectos del mencionado giro espacial y profundizamos en la producción del espacio desde las alternativas económicas apuntadas. Luego, proponemos la constelación de conceptos geográficos como catálogo teórico a partir del cual se toman las nociones de territorio y lugar. Así, describimos las principales dimensiones de ambos términos apoyándonos en un par de estudios de caso vinculados al ámbito rural de la provincia de Mendoza. Finalmente ensayamos algunas reflexiones y conclusiones sobre los diferentes aspectos teóricos y prácticos que intervienen en la elección de conceptos geográficos.
\end{abstract}

PALABRAS CLAVE: ESPACIO. TERRITORIO. LUGAR. ECONOMÍA SOCIAL Y SOLIDARIA.

\section{Territory and place: the spatiality under debate in the Social and Solidarity Economy. Conceptual approaches based on concrete rural experiences}

\begin{abstract}
In recent decades, we attend the so-called spatial turn in the social sciences. This is an ongoing process where the spatiality of social phenomena acquires great relevance for different disciplinary approaches. However, as researchers we are constantly faced with the challenge of choosing geographical concepts that allow us to explain
\end{abstract}


our objects-subjects of analysis. This text seeks to share this theoretical dilemma, focusing on the concepts of territory and place as possible analytical tools for the study of associative productive processes within the framework of the Social and Solidarity Economy. To do so, we develop some aspects of the mentioned spatial turn and deepen the production of space from the economic alternatives pointed out. Then, we propose the constellation of geographical concepts as a theoretical catalogue from which the notions of territory and place are taken. Thus, we describe the main dimensions of both terms based on a couple of case studies linked to the rural context of the province of Mendoza. Finally, we present some considerations and conclusions on the different theoretical and practical aspects involved in the choice of geographical concepts.

KEYWORDS: SPACE. TERRITORY. PLACE. SOCIAL AND SOLIDARITY ECONOMY.

PALAVRAS-CHAVE: ESPAÇO. TERRITÓRIO. LUGAR. ECONOMIA SOLIDÁRIA.

\section{Introducción}

El lenguaje coloquial está plagado de alusiones al espacio y otros términos geográficos, ya sea para recurrir al origen de una expresión, describir una determinada situación o establecer puntos de comparación con elementos conocidos, entre otros fines. Por ejemplo, Horacio González (2016) nos recuerda algunas de estas referencias específicamente en el plano político, partiendo de la división básica entre derecha e izquierda cuyo nacimiento se remonta a la ubicación de los/as deliberantes en uno u otro lado del parlamento. En Argentina, la tercera posición es un popular espacio político equidistante tanto de la izquierda como de la derecha. Por otro lado, analistas en temas políticos aseguran indagar en una determinada situación desde el llano cuando quieren destacar una mirada cercana a los acontecimientos en cuestión. Asimismo, en época de comicios, no faltan candidatos y candidatas que se jactan de haber bajado a los territorios con el objeto de resaltar su cercanía a los problemas cotidianos de los/as habitantes de la jurisdicción en donde compiten electoralmente.

Así como sucede en la política, de manera general en las ciencias sociales existen innumerables referencias a lo espacial como recurso metafórico o vinculación directa con aspectos geográficos de determinados objetos-sujetos de análisis. Si bien no se trata de algo novedoso, durante las últimas décadas ha aumentado el número de estudios que incluyen el enfoque espacial como tópico relevante. Conocido como el giro espacial (Soja, 1997) en las ciencias sociales, se trata de un proceso en marcha donde las categorías espaciales pasarían a dominar o equiparar a las del tiempo, contrarrestando una relación previa en que la temporalidad dominaba el quehacer en esta área del conocimiento.

El ámbito de las prácticas y los estudios sobre alternativas económicas al capital ${ }^{1}$ no ha sido ajeno a este proceso de espacialización. En tanto procesos sociales desarrollados durante las últimas décadas en todo el mundo y en Latinoamérica bajo características

1 El uso del término alternativas y sus derivados se presta para un sinnúmero de equívocos. En este sentido, "no es lo mismo una propuesta, un medio, un instrumento alternativo para resolver un problema especíico, que una sociedad alternativa que tiene por objetivo subvertir la existente (Etxezarreta, 2014:11). La Economía Social y Solidaria se mueve justamente entre estas variantes; en tanto proceso transicional, parte desde el empleo de instrumentos alternativos hacia el objetivo - de largo aliento- de alcanzar una sociedad distinta. La alternativa, en todo caso, puede entenderse en el mismo "proceso de lucha y de transformación, un proceso que se tiene que ir construyendo en la vida cotidiana, en la lucha por una sociedad diferente..." (Etxezarreta, 2014:12). 
particulares, variadas experiencias enmarcadas en la denominada Economía Social y Solidaria (ESS) ${ }^{2}$ han sido analizadas desde una mirada geográfica (Jurado y Cardozo, 2016; Altschuler y Pastore, 2015; Pinto de Godoy, 2010; Rincón Gamba, 2012; Zanca, 2007). Sin embargo, queda aún mucho terreno analítico por explorar no sólo a la hora de la comprensión de los respectivos contextos de su surgimiento, sino también en la identificación del espacio geográfico construido hasta el momento por los sujetos colectivos intervinientes en dichos procesos.

El presente trabajo es fruto de algunas reflexiones epistemológicas y metodológicas desarrolladas durante los últimos años, en el marco de diversos análisis en torno ESS y espacio geográfico. Específicamente, estas consideraciones teóricas surgen a partir del dilema por la elección de las categorías geográficas más adecuadas para dar cuenta de este tipo de experiencias socio-económicas. En particular, nos preguntamos por la utilidad de los conceptos de territorio y lugar, dos nociones de gran fuerza analítica y explicativa dentro de la Geografía. La elección de uno u otro concepto, ¿depende de la naturaleza del objeto-sujeto de análisis? ¿O más bien es una decisión plena de quienes investigamos?

Para ello, recuperamos, en primer lugar, algunos aspectos del mencionado giro espacial en las ciencias sociales, a modo de preámbulo. Luego, ponemos de relieve la mirada espacial como clave de estudio académica y política para procesos sociales, en general, y para trayectorias empíricas en ESS, en particular. Acto seguido, realizamos una breve mención a la constelación de conceptos geográficos, de forma tal de dimensionar la diversidad de nociones espaciales disponibles para nuestras investigaciones. A partir de allí, centramos nuestra atención en el concepto de territorio -cuyo empleo en trabajos geográficos ha adquirido primacía durante el último tiempo en América Latina- colocando el acento en algunos de sus aspectos y su posible vinculación con la ESS. Luego, desarrollamos la noción de lugary sus diferentes dimensiones, teniendo en cuenta que también puede dar cuenta del desarrollo de alternativas económicas al capital, pero a partir de determinadas condiciones locales. Tanto para territorio como lugar, recurrimos a casos de estudio del ámbito rural. Finalmente, enunciamos algunas consideraciones a modo de conclusiones parciales, que puedan alimentar nuevas reflexiones sobre el uso de conceptos espaciales para el estudio de diferentes procesos alternativos al capital.

\section{La geograficidad en debate: consideraciones sobre el giro espacial en las ciencias sociales}

El reconocido giro espacial en las ciencias sociales acaecido en la década de 1970 puede considerarse como un capítulo más en la relación entre historia y geografía. Según Piazzini (2006), la relación tiempo-espacio ha mutado de acuerdo a las diferentes épocas históricas. Así, durante el Medioevo, ambos términos eran inseparables, es decir, "el cuándo estaba casi universalmente conectado al dónde" (Giddens, 1994:29, en Piazzini, 2006:54). En cambio, con el advenimiento de la Modernidad, "el control político del espacio se hizo posible mediante el ordenamiento del tiempo" (Piazzini, 2006:54). 
Europa se erigía a sí misma como sinónimo de progreso y evolución, a la vez que ubicaba a América, Asia y África como realidades de un tiempo pretérito asociadas linealmente al atraso (Dussel, 2005). Según esta mirada colonial, la distancia geográfica entre esos mundos se correspondería a diferentes estadíos en una línea evolutiva que se dirigiría, inexorablemente, al modelo de desarrollo occidental. En otras palabras, "vivir en otra parte, en la periferia del mundo occidental, era vivir en otra época, pero no sólo en otra época, sino en un estado sociocultural de antecedencia evolutiva, agotado ya, o superado por la historia occidental" (Piazzini, 2006:54). El pensamiento moderno, tanto en su versión conservadora como crítica, le otorgaba al tiempo el papel ordenador de las relaciones sociales, relegando al espacio a un rol de contenedor o escenario de estas.

Sin embargo, como dijimos, desde la década de 1970 el cronocentrismo que primaba en las ciencias sociales hasta entonces le cede relevancia a la dimensión geográfica. El giro espacial toma forma y asistimos a una forma distinta de pensar e imaginar la(s) geografía(s). Según Soja (2010:182), se trata de un viraje sin precedentes en el cual diferentes autores y autoras han comenzado a interpretar el espacio y la espacialidad de la vida humana con la misma perspectiva crítica e interpretativa dada al tiempo y a la sociedad. En otras palabras, este giro implica la ponderación de tres términos en cualquier fenómeno humano: la historicidad, la socialidad y la espacialidad. La "trialéctica del ser", como la llama el autor, implica el estudio de manera entralazada y combinada de estas múltiples perspectivas (Soja, 2010:185).

El giro espacial en las ciencias sociales y los diferentes aspectos involucrados en él no pueden entenderse sin considerar los aportes realizados por Henri Lefebvre (Soja, 2010; Harvey, 2015; Martínez, 2014). Sus contribuciones en este sentido fueron variadas y se condensaron particularmente en la obra La Producción del Espacio (2013) ${ }^{3}$ donde el eje reside precisamente en considerar al espacio (social) como producto (social). Si bien esta afirmación se encuentra próxima a la tautología, no lo es tanto considerando que aún hoy existen miradas sobre el espacio que lo asocian a un mero continente de objetos y de relaciones sociales, centrándose únicamente en la ubicación de estos. En este último caso, siguiendo a Harvey (2015:128, traducción del autor), estaríamos en presencia de un tipo de espacio, el absoluto, que "se convierte en una 'cosa en sí misma', con una existencia independiente de la materia. Tiene entonces una estructura que podemos utilizar para clasificar o distinguir los fenómenos". Y agrega que, "socialmente, es el espacio de la propiedad privada y de otras entidades territoriales delimitadas (como Estado, unidades administrativas, planos urbanos y redes urbanas)" (Harvey, 2015:128, traducción del autor). Con esto, no descartamos la utilidad de este tipo de enfoque sobre el espacio, pero sí consideramos que el espectro de análisis debe ampliarse.

En este sentido, según Lefebvre (2013:86), "el espacio contiene relaciones sociales y es preciso saber cuáles, cómo y por qué”. Es decir, no sólo puede entenderse al espacio en tanto absoluto, sino también es preciso tomar en cuenta su condición de espacio relativo, "comprendido como una relación entre objetos que existe por el propio hecho de que los objetos existen y se relacionan", y de espacio relacional, es decir, "espacio (...) estando contenido en objetos, en el sentido de que un objeto puede ser considerado como existiendo solamente en la medida en que contiene y representa en sí mismo las relaciones con otros objetos" (Harvey, 2015:128, traducción del 
autor). Espacio absoluto, relativo y relacional debieran considerarse en simultáneo y entrelazados, aunque, según el caso, pueda centrarse la atención en una de esas dimensiones. De cualquier manera, la tarea consiste en una inversión en el análisis de los espacios, pasando de su condición de productos dados (estudiados de forma general, descritos, enumerados) a la producción de los mismos. Para ello, resulta indispensable acompañar dicho estudio geográfico por uno de tipo histórico que tome nota de las vicisitudes de los procesos.

Como se puede captar, el espacio no es un ente estático, sino que en su esencia se hallan el movimiento, la contradicción y la transformación. En este sentido, la propuesta de Milton Santos (2006) representa una de las aproximaciones más certeras a esta cuestión, al afirmar que el espacio es un conjunto de elementos fijos y flujos, donde los primeros, fijados en cada lugar, permiten acciones que modifican ese lugar. Por su parte, los flujos son resultado directo e indirecto de las acciones, atravesando los elementos fijos o instalándose en ellos, modificando así su significado y su valor, a la vez que son alterados también por este proceso. El mismo autor agrega que el espacio está formado por "un conjunto indisociable, solidario y también contradictorio de sistemas de objetos y sistemas de acciones, no considerados separadamente sino como el cuadro único en el cual la historia se da" (Santos, 2006:39, traducción del autor).

Así, la naturaleza de un espacio determinado dependerá en gran parte de las relaciones sociales que allí imperen, que a su vez estarán condicionadas por el modo de producción vigente: "cada sociedad (en consecuencia, cada modo de producción con las diversidades que engloba, las sociedades particulares donde se reconoce el concepto general) produce un espacio, su espacio" (Lefebvre, 2013:90). Para poder descifrar esa compleja trama detrás del proceso de producción de un espacio dado, se precisa tener en cuenta tres dimensiones que, como parte de un proceso dialéctico mayor, son distintas y complementarias y están tensionadas a la vez, según el lugar, el momento y el modo de producción en cuestión. Por un lado, encontramos a la práctica espacial que incluye producción y reproducción, lugares específicos y conjuntos espaciales propios de cada formación social y que asegura una determinada continuidad social de manera más o menos cohesionada. La segunda dimensión consiste en las denominadas representaciones del espacio, es decir, el espacio concebido vinculado a las relaciones de producción imperantes y el orden que aplican. La tercera dimensión, los espacios de representación o espacio vivido, expresan simbolismos complejos ligados al lado clandestino y subterráneo de la vida social (Lefebvre, 2013:92-97). Esta propuesta analítica tiene la fortaleza de combinar lo abstracto y lo material del espacio, por medio de una relación dialéctica entre lo percibido (práctica espacial), lo concebido (representaciones del espacio) y lo vivido (espacios de representación), por lo que "las relaciones entre esos tres momentos no son nunca simples ni estables" (Lefebvre, 2013:104). Mientras que las representaciones del espacio, desde lo abstracto de la planificación vertical $a$ priori, afirman la reproducción del capital, la última dimensión expresa -desde las diversas identidades de clase, género, étnicas, sexuales, entre otras- contra-espacios o espacios de resistencias basados en formas de conocimientos locales y menos formales (Oslender, 1999).

Sin embargo, bajo la preeminencia mundial del modo de producción capitalista, la producción del espacio está fuertemente condicionada por las metas que se proponen los actores sociales que dominan la escena político-económica. Por ello, el espacio 
concebido por el capital toma mayor relevancia en este proceso. En este sentido, el capitalismo ha logrado escindir la producción y el dominio del espacio, por un lado, y la apropiación, por otro. Como afirma Martínez,

(...) el hombre ya no es lo que produce, su yo exteriorizado no es reconocible en el mundo objetivado de las cosas (...) Hay dominación pero no apropiación, que daría sentido a todo el proceso, al reconocerse el hombre en la obra creada y en el Otro". (Martínez, 2014:4).

En cambio, el espacio vivido por el sujeto es, ante todo, su espacio de acción e imaginación (Martínez, 2014:17). Se trata de la apropiación del espacio-lugar, el cual refiere al hecho físico de la ciudad o el campo, y al conjunto de significaciones y relaciones implicadas en la vida social en esos ámbitos. Es espacio material y simbólico a la vez. Dicho de otro modo, la apropiación espacial implica conjunto de prácticas sociales que confieren a un espacio determinado las cualidades de un lugar, de una obra (Martínez, 2014:2). Asimismo, muchas de esas prácticas sociales se llevan a cabo a diario por los sujetos. Sin embargo, esa "cotidianidad no es sólo el espacio-tiempo donde se encuadra todo lo insignificante, sino que es presentado como la instancia transcendente donde efectuar la apropiación del mundo por parte del hombre, ese homo quotidianus" (Martínez, 2014:6, cursiva del autor). Es aquí, en estos espacios vividos y apropiados a partir de la cotidianidad, donde podemos ubicar a las experiencias socio-productivas alternativas a la lógica del capital.

\section{La producción del espacio desde las alternativas al capital}

Como dejamos entrever algunas líneas atrás, bajo la preeminencia mundial del capitalismo, la producción del espacio está fuertemente condicionada por las metas que se proponen los sujetos que actúan según su lógica y dominan la escena político-económica. Por ello, el espacio concebido por el capital toma mayor relevancia en este proceso. En cambio, la apuesta por prácticas y proyectos políticos que se distancien de la lógica del capital supone imaginar y producir otro espacio. En este sentido, el movimiento intelectual y práctico (político) partiría desde la identificación del espacio vivido propio de las resistencias hacia la creación y recreación de experiencias concretas que construyan espacios propicios para pensar la transformación social.

Pero, antes de proseguir, resulta indispensable explicitar algunos lineamientos sobre lo que se entiende por ESS. Por un lado, se trata de la denominación de un amplio espectro de prácticas dedicadas a la producción, comercialización y consumo de bienes y servicios. Estas trayectorias empíricas poseen en común la ausencia de apropiación privada de los excedentes generados por sus actividades, basándose en mecanismos de trabajo caracterizados por el asociativismo y la autogestión. Son actividades socioeconómicas que apuntan, sobre todo, a la reproducción de la vida de quienes se involucran en ellas y de su entorno próximo -naturaleza incluida-. En términos específicos, podemos ensayar un catálogo de experiencias concretas entre las que encontramos a empresas recuperadas por sus trabajadores/as, organizaciones sociales del campo y la ciudad, unidades asociativas de baja escala de producción (familiares o barriales), ferias populares, clubes de trueque, grupos de productores/as que trabajan bajo los principios de la agroecología, entidades distribuidoras de productos, mutuales, 
asociaciones, cooperativas de trabajo y de otros tipos. A este listado también se pueden agregar prácticas educativas que se organicen bajo los principios del cooperativismo a la vez que lo promuevan, como experiencias de salud comunitaria, centros culturales y clubes barriales, entre otras.

Por otra parte, mediante esta denominación (y otras similares) 4 se hace referencia al proyecto político en construcción que pretende emanciparse de la lógica de la acumulación capitalista. Con diferentes grados de radicalidad, se trata de la edificación de un proceso transicional hacia una sociedad donde prime el trabajo (y con ello, los/ as trabajadores/as) por sobre el capital. Hablar de ESS implica entonces una referencia a procesos socio-económicos en marcha que, mientras sortean las dificultades impuestas por el capitalismo, prefiguran una sociedad distinta a la predominante. Es decir, consiste, por un lado, en lo realmente existente (presente) y, por el otro, en lo proyectado (futuro).

Retomando los aspectos geográficos, la construcción de espacios que posibiliten la producción, la distribución y el consumo de productos (y servicios), identificando necesidades y capacidades (locales y regionales) para satisfacerlas, se presenta como un desafío permanente para las organizaciones de la ESS. La situación desventajosa que suelen enfrentar estas experiencias, en comparación a otras similares del campo de la economía capitalista, las obliga a resistir en muchos casos. Estas dificultades suelen tomar cuerpo en la escasez de capital para adquirir equipamiento y/o contar con materia prima para comenzar procesos productivos. En numerosas ocasiones, esa ausencia de capital necesario imposibilita la obtención de habilitaciones legales, lo cual las empuja a desempeñar sus tareas bajo cierta informalidad, desenvolviéndose entre pregones en determinados ámbitos y el ocultamiento en otros. Por ende, el desafío al que se enfrentan es doble; por un lado, identificar espacios vacantes dejados por el capital donde puedan nacer, resistir y desarrollarse bajo los principios de la ESS; y por el otro, imaginar y concretar nuevos espacios que, aún a pequeña escala, se alimenten de lo vivido de las alternativas, buscando afectar el espacio concebido.

En definitiva, de lo antedicho se desprende que la lógica del capital, a través de determinados sujetos -las empresas del capital-, comandaría la producción del espacio buscando dominar o excluir aquello que se le contraponga. En cambio, las alternativas a esa lógica -aquí las experiencias concretas de la ESS- resistirían (y, en la media de lo posible, avanzarían) en esos espacios bajo otras lógicas de apropiación. Nuestra atención se dirige entonces al análisis de esta última lógica y de sus formas de producir espacio.

\section{Una constelación de conceptos geográficos}

El interés general por dar cuenta de procesos enmarcados en la ESS a través de un análisis espacial encierra, como ya se anticipó, algunos dilemas académicos. Particularmente hacemos referencia al momento de definir aquellas nociones geográficas que permitan llevar a cabo esa tarea intelectual, lo que no suele ser una labor sencilla y libre de contradicciones. Se trata de una decisión que inevitablemente lleva consigo una serie de criterios epistemológicos, metodológicos y políticos.

4 Nuevo Cooperativismo, Economía del Trabajo, Economía Popular y Solidaria, entre otras denominaciones. 
Una posible respuesta a este dilema la podemos hallar en la propuesta de Haesbaert (2014) en torno a la constelación de conceptos geográficos. La misma contendría una categoría - "una especie de 'concepto más amplio' o más general”- desde donde se desprenden una serie conceptos (Haesbaert, 2009, traducción del autor). En geografía, según el autor brasilero,

(...) podemos proponer el "espacio" como una categoría, nuestro concepto más general, y que se impone frente a los otros conceptos - región, territorio, lugar, paisaje, [escala, ambiente]... Estos formarían la "constelación" o conceptos geográficos "familiares" (como prefería Milton Santos). (Haesbaert, 2014:22, traducción del autor).

Todos ellos constituirían la constelación geográfica de conceptos; cada concepto alumbra un aspecto específico de la realidad siempre bajo la influencia del espacio como noción central. Vale aclarar que cada concepto es siempre heterogéneo y nunca puede ser tratado de forma aislada. Justamente la idea de la constelación posibilita entender a cada noción en un marco mayor, rodeada de otras (Haesbaert, 2009).

Ahora bien, en la elección de los conceptos más adecuados para llevar a cabo nuestros análisis reside uno de los desafíos más importantes para quien investiga. Es allí también donde se despliega una de las habilidades propias de este oficio. Este dilema puede ser expresado de la siguiente forma:

En cierto extraño sentido, me siento como si estuviera paseando por un hermoso jardín multidimensional, escogiendo un camino intelectual particular para llenar mi cesta de encantadoras flores. Pero, téngase esto en cuenta, al escribir este ensayo me veo forzado a seguir sólo un camino de los muchos posibles, y en otra ocasión podría escoger una ruta diferente y flores distintas para la cesta. Como geógrafos sabemos que el mapa que creamos depende de la proyección que utilicemos y todas las proyecciones necesariamente van a distorsionar la verdad de algún modo (Gould, 1987).

En este caso, Gould pone de relieve el rol activo de quien investiga a la hora de la elección de la ruta a seguir, como así también reconoce que esa decisión afectará al objeto de estudio. Por su parte, Saquet (2014), de manera más esquemática, sostiene que la elección obedecerá a nuestra orientación teórico-metodológica (cuestión epistemológica), al objeto-sujeto de estudio y del componente de lo real (cuestión ontológica) y a nuestra orientación política (cuestión de la praxis). Es decir, la elección de la/s categoría/a sería fruto de una reflexión que combine estos tres aspectos de la investigación.

En términos más concretos, de lo que se trata es de definir el foco de nuestra investigación lo cual permitirá identificar el concepto a emplear, sin que ello implique renunciar a otros conceptos; la frontera entre uno y otro concepto puede ser difusa. Como sostienen Haesbaert

(...) la ventaja en este caso es que cuando hablamos de "enfoque", al mismo tiempo que hacemos hincapié en una determinada perspectiva o forma de mirar, de abordar nuestra pregunta u "objeto" (...), no ignoramos las demás 
posibilidades de "enfoque" -que, aunque "desenfocadas", siguen estando presentes allí (Haesbaert, 2009:8, traducción del autor).

Ahora bien, en este trabajo hemos decidido tomar a la ESS para poner en debate la inquietud por la elección de conceptos geográficos como herramientas de análisis. Para ello, en los párrafos que siguen mencionamos dos experiencias concretas cuyas características comunes son las siguientes: i) desarrollan sus actividades en el ámbito rural de la provincia de Mendoza (Argentina), ii) ambas son parte de la misma organización campesina y iii) la producción de alimentos es un aspecto esencial para ellas. En términos geográficos, el ejercicio que planteamos discurre alrededor de la elección de dos conceptos integrantes de la constelación mencionada: territorio y lugar. A priori, nos preguntamos: ¿qué herramientas analíticas ofrece cada concepto? ¿Qué consideraciones entran en juego para la elección de uno u otro concepto? ¿Acaso es el problema de nuestra investigación lo que define el foco a realizar en la constelación? ¿O más bien se trata de motivaciones políticas sobre esta temática? ¿Puede suponerse que las mismas trayectorias empíricas a analizar inviten a la elección de una u otra noción?

\section{Territorio, una posibilidad}

En el marco del giro espacial en las ciencias sociales, el concepto de territorio ha sido uno de los más empleados de la mencionada constelación durante las últimas décadas. Esta noción se ha convertido en una herramienta analítica muy utilizada para el estudio de diferentes disputas sobre el espacio. En particular, el concepto ha servido para el análisis de diversos movimientos sociales en América Latina donde es central para las bases de la teoría y la práctica política (Halvorsen, Fernandes y Torres, 2019:1455). En los párrafos que siguen, mencionaremos las principales dimensiones de este concepto y sus implicancias, aplicando alguna de ellas a un caso de estudio sobre un conflicto de tierras en el sur de la provincia de Mendoza.

\section{El territorio como poder materializado}

Cuando diferentes sujetos sociales con divergentes miradas políticas se enfrentan por el destino de un determinado espacio, un movimiento posible dentro de la constelación de conceptos geográficos puede dirigirse hacia la noción de territorio. De manera general, este concepto refiere a "un espacio definido y delimitado por y a partir de relaciones de poder” (Lopes de Souza, 2012:78, traducción del autor). Como ya anticipamos, en el marco del mencionado giro espacial, se trata de un concepto cuyo uso ha sido recurrente a la hora de analizar diversos procesos sociales. De allí que muchas veces se prefiera hablar de un giro territorial, resaltando el valor que ha adquirido esta noción en las últimas décadas. En el caso latinoamericano, su empleo se extendió, principalmente, de la mano de las valiosas contribuciones de la geografía brasilera (Porto Goncalves, 2009; Saquet, 2013; Fernandes, 2009; Haesbaert, 2004; Lopes de Souza, 2012; entre otros/ as) que renovaron aquel enfoque centrado en el territorio como uno de los componentes del Estado-nación moderno para ampliarlo al estudio sobre otros fenómenos sociales (entre los que se destacan los análisis vinculados a movimientos sociales).

Sin embargo, la renovación del concepto de territorio no solo se dio en América Latina, sino también en el ámbito anglosajón (Elden, 2013; Raffestin, 1993; Sack, 1986). 
En este sentido, Sack señala que junto al concepto territorio, se encuentra la idea de territorialidad, es decir, "el intento por parte de un individuo o grupo de afectar, influenciar o controlar personas, fenómenos y relaciones, a través de la delimitación y el establecimiento de un control sobre un área geográfica" (Sack, 1986:1). Se trata de un aspecto inherente a la naturaleza de los grupos sociales, dado que indefectible precisan del control de un espacio para su reproducción. Por ende, la territorialización es el acto, la acción o el movimiento a través del cual un espacio es apropiado y dominado (Haesbaert, 2007). Es decir, es el mecanismo por medio del cual se produce territorio. Asimismo, la concepción de este término ha sido enriquecida en las últimas décadas abriendo un abanico de significados y, por ende, de usos. Por ejemplo, existen enfoques idealistas que enfatizan la dimensión simbólica expresada en representaciones por parte de un determinado grupo social; Bonnemaison y Cambrèzy (1996, en Haesbaert, 2004) trabajan con una concepción cultural del territorio, ya que, según su mirada, antes de ser una materialidad, es un valor. Por ende, el territorio tendría un carácter más simbólico -asociado a cierta inmaterialidad- que concreto. Se trata de un aporte valioso que amplía los márgenes analíticos de las relaciones de poder espaciales. A modo de síntesis, Torres ensaya una definición para abarcar estas diversas miradas: "el territorio se entiende como la manifestación espacial del poder fundamentada en relaciones sociales determinadas, en diferentes grados, por la presencia de energía -acciones y estructuras concretas- y de información -acciones y estructuras simbólicas-" (Torres, 2011:212).

Más allá de la dimensión simbólica que queda en evidencia en las últimas consideraciones, no renunciamos a la consideración de la materialidad que este concepto conlleva: "todo territorio, geográficamente hablando, tiene siempre una base espacio-material para su constitución” (Haesbaert, 2013). Esta afirmación encuentra su fundamento si se considera que el espacio, en tanto categoría esencial de la constelación de conceptos mencionada, cuya naturaleza envuelve al territorio (alumbra), presenta -como lo hace Milton Santos- un "carácter material y evidente" (Zusman, 2002:210). En resumen, materialidad y subjetividad (inmaterialidad) son aspectos constituyentes del territorio.

\section{El territorio en las alternativas al capital: lógica campesina vs. lógica empresaria}

Como señalamos más arriba, es indudable la fuerza analítica e interpretativa que posee, a priori, el concepto de territorio a la hora de analizar relaciones de poder bajo un enfoque geográfico. En este sentido, el estudio de alternativas económicas al capital podría ser - por una cuestión ontológica- materia de análisis a través de esta noción, dada la naturaleza subalterna y disruptiva de las mismas respecto a las formas económicas dominantes. Es decir, en muchos casos los sujetos de la ESS se relacionan de manera conflictiva con el Estado y el capital en el espacio, “disputándose la apropiación y el control de recursos a partir de concepciones y prácticas espaciales diferentes y en ocasiones opuestas, que responden a lógicas económicas y formas de significar distintas" (Rincón Gamba, 2012:25). Cada uno de estos sujetos actúan bajo lógicas políticas y económicas distintas, por lo que cada uno "territorializa el espacio bajo fines y estrategias distintas” (Rincón Gamba, 2012:25).

En el caso de los sujetos de la ESS, esto se tornaría aún más evidente en aquellos movimientos sociales-territoriales para los cuales el territorio es su triunfo y, por 
lo tanto, la razón de su existencia (Fernandes, 2005). Son organizaciones sociales rurales o urbanas que encuentran en el territorio, el objeto central de su lucha y la clave para alcanzar sus objetivos y metas. A la vez, esta noción permite informar sobre la identidad de las mismas generando nuevas subjetividades políticas en el curso de la movilización. El territorio, en estos casos, se convierte en un sitio de socialización política (con resultados materiales e inmateriales) y, a través de procesos de territorialización-desterritorialización-reterritorialización, las organizaciones involucradas institucionalizan sus prácticas ante otros sujetos (particularmente el Estado) (Halvorsen et al., 2019:1456). Visto desde las mismas organizaciones, se vislumbra, por un lado, solidaridades a la hora de la apropiación de ese territorio y, por el otro, contradicciones y disputas frente a otros sujetos que buscan controlarlo. En relación con este último aspecto, las disputas por el territorio pueden ser analizadas en clave de conflicto, entendido como

(...) el estado de enfrentamiento entre fuerzas opuestas, relaciones sociales distintas, en condiciones políticas adversas, que buscan por medio de la negociación, de la manifestación, de la lucha popular, del diálogo, la superación, que se da con la victoria, la derrota o el empate (Fernandes, 2004:18).

A su vez, existe otra noción fundamental para entender este tipo de disputas. Se trata de la idea de conflictualidad, la que consiste en una propiedad de los conflictos. La conflictualidad promueve, en términos amplios, la transformación de territorios, modificando paisajes, creando comunidades, empresas y municipios, cambiando sistemas agrarios y bases técnicas, complementando mercados, rehaciendo costumbres y culturas, reinventando modos de vida, reeditando permanentemente el mapa de geografía agraria y reelaborado por diferentes modelos de desarrollo (Fernandes, 2004:4). Así, mientras el conflicto puede ser resuelto, la conflictualidad no, ya que "esta es producida y alimentada día a día por el desarrollo desigual del capitalismo" (Fernandes, 2004:18) Si bien el autor brasilero ensaya estos enunciados para el caso de la problemática rural y los movimientos campesinos, no resulta inadecuado utilizar sus apreciaciones para el análisis de organizaciones rurales y urbanas de la ESS.

Llegados a este punto, recurrimos a un conflicto, a modo de ejemplo, para ensayar un diálogo entre el caso y las dimensiones del concepto de territorio. Se trata del trabajo titulado Territorialidad y resistencias campesinas: el conflicto de "Los Leones" (Mendoza, Argentina) (Liceaga, 2017), que versa, como su nombre lo indica, sobre una disputa de tierras entre pequeños/as productores/as rurales organizados/as en un movimiento campesino y un grupo de empresarios que buscaban el control de espacios comunitarios mediante su cercamiento. La disputa en cuestión comenzó en 2006, cuando un grupo de familias campesinas que habitaban en el paraje Los Leones en el Departamento de San Rafael (sur de Mendoza) se organizó frente a la amenaza de perder sus tierras. Esa coordinación fue en torno a la Unión de Trabajadores Rurales sin Tierra (UST), organización campesina de base integrada por más de 500 familias de trabajadores/as rurales, campesinos/as e indígenas, presente en diferentes departamentos de la provincia de Mendoza y en parte de la provincia de San Juan. Nacida en 2001, la organización se divide espacialmente en grupos de base y, temáticamente, en áreas de trabajo. Su sede se halla en Jocolí, localidad perteneciente al Departamento de Lavalle (norte de Mendoza), donde cuenta con el mayor número de grupos de base. Como integrante del Movimiento Nacional Campesina Indígena (MNCI), la UST "levanta las 
banderas políticas" de la reforma agraria integral, la defensa de los territorios campesinos, la organización popular, la soberanía alimentaria de los pueblos (Liceaga, 2008).

En este caso, la organización se involucró en un definido conflicto de tierras. Como su autor señala, se trató del enfrentamiento entre una lógica territorial campesina construida por décadas que incluye no sólo aspectos económicos, sino también culturales, y otra concebida por el capital, encarnada en un grupo de abogados-empresarios, cuya expresión material más evidente ha sido el cercamiento de tierras destinadas a la cría de vacunos (Liceaga, 2017). A modo de diálogo entre el caso y lo dicho en este escrito hasta el momento, podríamos apuntar -de manera esquemática- que la lógica territorial campesina expresaba más firmemente aspectos del espacio relativo y relacional, mientras que la lógica del capital reflejaba dimensiones del espacio absoluto (cercamiento y delimitación del espacio). En términos específicos, según el mismo autor, el "concepto de territorio/territorialidad se mostró como una contraparte adecuada, en la medida en que ayudó tanto a circunscribir determinados escenarios empíricos para la investigación, como a considerar las diversas dimensiones que confluyen en la acción social" (Liceaga, 2017:241). A su vez, a lo largo del análisis del conflicto, se llevó a cabo una "articulación conceptual entre territorio-territorialidad y campesinado" que posibilitó estudiar una pluralidad de situaciones y aspectos de lo real (Liceaga, 2017:241). En este sentido, detectamos un vínculo estrecho entre lo dicho respecto a los movimientos socio-territoriales y el ámbito campesino. El análisis del conflicto "acentuó y puso de relieve la inherente politicidad que impregna la construcción de la territorialidad campesina" en un marco general -conflictualidad- entre la lógica del agronegocio y la producción campesina (Liceaga, 2017:241).

Visto este conflicto desde el plano productivo, los interrogantes que surgen a partir de un posible triunfo por parte del sujeto subalterno (los/a campesinos/as) en la disputa buscan develar los usos que se le darán a estas tierras:

¿Se está ante un aumento de la mercantilización de las actividades y, consecuentemente, de una mayor dependencia? ¿Es la capitalización y el aumento de la presencia en los mercados tradicionales la única alternativa posible para los campesinos? En un contexto histórico en el que la Reforma Agraria Integral sólo puede plantearse como una dimensión utópica de la praxis ¿existe alguna vía alternativa para el campesinado, que no sea su conversión en "pequeños productores"? La respuesta a estas preguntas es difícil y en última instancia no puede darse sin que pese una fuerte valoración política. La experiencia de Los Leones muestra, no obstante, que la Economía Social es un camino posible para el fortalecimiento del campesinado, la creación de mercados no dominados completamente por el gran capital y la articulación con otros sujetos económicos y políticos (Liceaga, 2017:243).

Según el autor, los circuitos productivos diseñados desde la Economía Social (que en este trabajo hemos preferido llamar ESS) pueden ser vías alternativas para un sector del campesinado fuertemente condicionado y asediado por la lógica mercantil, aspecto que en el caso citado ha alcanzado un nivel superlativo, al poner en peligro el control del espacio necesario para la producción. Justamente la noción de territorio aquí resulta pertinente para analizar este conflicto geográfico que, en el marco de una determinada conflictualidad, encierra dos lógicas distintas: por un lado, la defensa de los/as 
campesinos/as de una porción de tierra indispensable para su sobrevivencia; y por el otro, el avance de un grupo empresarial sobre un espacio que aún no está plenamente integrado a la lógica del capital.

Dándole una interpretación más a este caso desde la ESS, podemos razonar que, si mediante la producción campesina se apuesta a colocar alimentos sanos y accesibles para las comunidades cercanas, la problemática en torno al acceso a la tierra (y al agua, en el caso de ambientes áridos como la provincia de Mendoza) resulta una realidad insoslayable. Es decir, el acceso a determinados territorios (o la resistencia en el caso de los ya ocupados) se convierte en un vehículo impostergable para alcanzar el objetivo de la producción de alimentos. Sin tierra no hay cultivos y sin ellos no hay materias primas y alimentos para volcar a los circuitos productivos solidarios. Visto así, este desafío conecta a la ESS con la consigna de reforma agraria integral propia de los movimientos campesinos.

Vale aclarar que, así como la disputa por el territorio en el ámbito campesino resultaría esencial para el devenir de proyectos y acciones en el marco de la ESS, en contextos urbanos también lo serían: ocupaciones de fábricas por parte de trabajadores y trabajadoras, ferias de la economía popular en espacios públicos que resisten ante la amenaza de desalojo, clausura de centros culturales barriales, entre otros.

Sin embargo, no siempre el territorio resulta ser razón de existencia y/o acciones de las organizaciones vinculadas a la ESS. Si bien en algunos casos, el debate en torno al control y el uso de un determinado territorio puede intervenir en la vida cotidiana de estas organizaciones, el eje de su actividad general puede no residir allí, ni su supervivencia depender de ese aspecto. En muchos casos se trata de sujetos que, de alguna manera, construyen un espacio específico despegándose de la lógica económica hegemónica. A pesar de esto, no existe un conflicto claramente marcado entre sujetos por la apropiación de un determinado espacio, aunque los usos que de él se hagan, contraríen el orden económico, social o político imperante. El campo de la ESS resulta amplio y diverso en cuanto a experiencias concretas en acción, por lo cual la mirada en tanto investigadores/ as puede posarse en diferentes aspectos de la espacialidad de estas prácticas. Volviendo a la propuesta metodológica y analítica en torno a la constelación de conceptos geográficos, será territorio si, como en este ejemplo, nos concentramos en las relaciones de poder (y en otros aspectos asociados a las mismas). Pero podrá ser también paisaje si nuestro foco se centra cuestiones de carácter más simbólico y cultural, ambiente para la relación sociedad-naturaleza o lugar para aspectos identitarios.

Precisamente a continuación desarrollamos algunas consideraciones en torno a la noción de lugar como otra llave posible de análisis para el estudio de experiencias concretas en ESS.

\section{Lugar, otra posibilidad}

Dentro de la variada gama teórica que nos brinda la mencionada constelación de conceptos geográficos, se halla la noción de lugar, susceptible de convertirse en una llave para analizar trayectorias empíricas en ESS desde una mirada distinta del conflicto espacial abierto. Por ello, a continuación, mencionaremos las principales 
Territorio y lugar: la espacialidad en debate...

EMANUEl ALberTo JURADO

dimensiones de este concepto y recurriremos, a modo de ejemplo, a una experiencia empírica de la ESS que, al igual que el ejemplo anterior, enmarca su trabajo en el ámbito rural de la provincia de Mendoza y es parte de la misma organización campesina.

\section{El lugar como expresión espacial de la cotidianidad}

La irrupción de la ESS en América Latina durante las últimas décadas motivó el nacimiento de numerosas experiencias concretas, muchas de las cuales pueden ser caracterizadas -a grosso modo- como pequeñas unidades productivas y/o comerciales. El estudio de estas nos ha planteado el dilema sobre los límites que este tipo de acciones -dado su tamaño- puede tener en cuanto herramientas transformadoras de una realidad que la excede largamente y condiciona fuertemente su desenvolvimiento. De lo que se trata es del histórico debate en el interior de las ciencias sociales entre estructura y sujeto, cuestión que tiene su correlato geográfico. El espacio entendido como producto de relaciones sociales y productor de las mismas (Lefebvre, 2013), obliga a poner el eje del análisis en el domino de esa producción. Bajo las condiciones actuales de preeminencia del sistema capitalista, la lógica predominante en la producción del espacio indudablemente se corresponde con la reproducción del capital. Así entendido, desde el punto de vista analítico, el espacio se constituye en una totalidad que presiona al sujeto. Sin embargo, existen numerosas y variadas experiencias que, desde su labor cotidiana, con fuerte arraigo local y basadas en determinados aspectos identitarios -sus espacios vividos-, contrarían esos designios del capital -los espacios concebidos-. Ante este panorama, podríamos situar a esas resistencias en el ámbito de los lugares (Oslender, 1999), concepto que permitiría referirse a los espacios conectados con la cotidianidad del sujeto y la reproducción de su vida (Hinkelammert y Mora Jiménez, 2005).

Antes de continuar con una descripción del concepto de lugar, es importante remarcar que la mención a lo local como ámbito de trabajo precisa de algunas consideraciones en torno a la noción de escala -componente de la constelación de conceptos mencionada- y que, según Lopes de Souza (2013), puede ser considerada bajo tres acepciones distintas pero relacionadas a la vez: "escala del fenómeno", "escala de análisis" y "escala de acción". Mientras la primera refiere a la naturaleza del objeto de investigación, la "escala de análisis"

(...) es intelectualmente construida como un nivel analítico, capaz de facultarnos a la aprehensión de las características relevantes de una cosa que estamos investigando o intentando descubrir, a partir de una pregunta o de un problema que tengamos formulado (Lopes de Souza, 2013:182, traducción del autor).

Por último, la "escala de acción (apunta a) un objeto específico y muy directamente político: aquello referente, en un raciocinio eminentemente estratégico, a la reflexión acerca del alcance espacial de los agentes" (Lopes de Souza, 2013:182, traducción del autor). En cualquiera de los tres casos, la escala no se presenta como una "categoría (para nosotros/as en este trabajo, concepto) pre-existente, apenas a la espera para ser aplicada, pero si un modo de contextualizar concepciones de la realidad" (Marston, 2004; en Lopes de Souza, 2013:191, traducción del autor). Sin embargo, parece razonable aceptar que, en principio, la escala local referiría a 
(...) recortes espaciales que, en grados variables, de acuerdo con su tamaño, expresan la posibilidad de una vivencia personal intensa del espacio (...) y, adicionalmente, la posibilidad de la formación de identidades socio-espaciales bastante particulares sobre la base de esa vivencia (Lopes de Souza, 2013:201, traducción del autor).

Pues bien, en este caso, el desarrollo de experiencias socio-productivas de la ESS a una escala local se sustentaría en un determinado arraigo en el campo y a partir de vínculos de proximidad entre quienes la integran. En otras palabras, la escala local generaría algún tipo de identidad en ese sentido -la comunidad campesina en este caso- por lo cual, siguiendo la sugerencia de Oslender (1999), resultaría susceptible de ser trabajada desde la noción de lugar.

Agnew (2011) ha realizado una de las propuestas analíticas más adecuadas para abarcar el estudio del lugar desde todas las dimensiones mencionadas. Al igual que Lefebvre, basa sus supuestos en un análisis fundado en tres ejes con el que se distancia de los análisis dicotómicos, para poder captar así la complejidad del fenómeno espacial:

La primera dimensión es el lugar como localización o un sitio en el espacio donde una actividad u objeto está localizado y que se relaciona con otros sitios o localizaciones por medio de la interacción, el movimiento y la difusión entre ellos (...) La segunda es la mirada del lugar como una serie de "locales" (localidades) o escenas donde las actividades diarias se desarrollan (...) La tercera dimensión es el lugar como sentido de lugar o identificación con el lugar como una única comunidad, paisaje y orden moral (Agnew, 2011:326, traducción del autor).

Como se puede constatar, la primera dimensión recupera el lugar en su cualidad de emplazamiento, aunque a la vez plantea un juego multiescalar; los mundos sociales locales (locale) del lugar no pueden entenderse por fuera del macro orden objetivo de la localización y la subjetividad del sentido de lugar (Agnew, 1987:3). Precisamente, este sentido de lugar "refuerza la definición socioespacial de lugar desde adentro, por así decirlo (y) en su diferenciación con respecto a otros lugares puede convertirse en un 'objeto' de identidad para un 'sujeto"' (Agnew, 1987:2). Según esta visión, se considera al lugar como un segmento del espacio cargado de significados, un espacio de la experiencia vivida (Cresswell, 2011). Evidentemente esta connotación retrotrae nuevamente al espacio vivido propuesto por Lefebvre como parte de la dialéctica del espacio, demostrando la necesidad de poner en consideración los aspectos subjetivos en la producción del espacio. Como señala Tuan, "lugar refiere al proceso por el cual la vida cotidiana está inscripta en el espacio y dotada de significado por grupos específicos de personas y sus organizaciones" (Tuan, 1977:164; en Hoelscher, 2011:252, traducción del autor).

Sin embargo, es necesario aclarar que esa diferenciación mencionada, no implica aislamiento, sino todo lo contrario: "la identidad de un lugar -cualquier lugar- no está arraigada simplemente dentro del lugar, sino que está compuesta también por relaciones externas" (Massey, 2004:79). Dicho de otra forma,

(...) la especificidad de cada lugar es el resultado de la mezcla distinta de todas las relaciones, prácticas, intercambios, etc. que se entrelazan dentro de este 
nodo y es producto también de lo que se desarrolle como resultado de este entrelazamiento. Es algo que yo he denominado "un sentido global de lugar", un sentido global de lo local (Massey, 2004:79).

Es decir, si bien es clave dar con estos significados específicos, los cuales están dados por las particularidades que encarna cada lugar, es importante aclarar que esa singularidad, no va en desmedro de vinculaciones con el afuera. Por el contrario, consideramos que el espacio cuenta con un componente relacional, por lo que ningún lugar existe salvo en relación con otros y cada uno contiene otros que están conectados con él. En este sentido, los aportes de Massey renuevan la mirada de Agnew y permiten comprender aún más la relación espacio-lugar. De todas maneras, es preciso dejar en claro que esas vinculaciones se dan bajo relaciones de poder. Es decir, en determinadas relaciones, un lugar puede tener una posición dominante, mientras que en otras tiene una posición más o menos subordinada (Massey, 2004: 82). Se trata de lo que la misma autora ha dado en llamar "geometrías del poder" (Massey 2008; 2004).

Teniendo en cuenta estos últimos aportes, es factible tomar al lugar como posible sede de las resistencias al espacio concebido de Lefebvre, donde la creatividad de los emprendimientos asociativos, empresas recuperadas, nuevas cooperativas y otras organizaciones de la ESS encuentran un terreno propicio para su surgimiento y desarrollo.

\section{El lugar como herramienta conceptual de aproximación a las alternativas al capital}

Al igual que el ejemplo utilizado en apartado anterior, aquí centramos nuestra atención en una experiencia concreta que forma parte de la UST. En este caso tomamos un trabajo propio titulado Aspectos socio-espaciales de experiencias económicas alternativas. La economía social y solidaria en Mendoza de 2001 a 2017: un estudio desde los casos de El Arca y la Unión de Trabajadores Rurales sin Tierra (Jurado, 2018), aunque, a diferencia el ejemplo ya analizado, nuestro interés se dirigió específicamente a conocer algunos aspectos espaciales del circuito productivo ideado por esta organización campesina. Es decir, en términos generales, el problema de investigación apuntó al trabajo realizado por el Área de Producción y Comercialización de la organización. Si bien para esta área la defensa de territorios o la conquista de nuevos resultan relevantes (por ejemplo, como posibles fuentes de materias primas para la producción), lo es de manera más o menos tangencial a su trabajo diario, ya que la energía de quienes trabajan en ella está principalmente centrada en la cotidianidad de la producción y comercialización (Jurado, 2018). Es decir, para estas trayectorias empíricas en ESS el control de un determinado territorio sólo aparece puntualmente como objeto de preocupación, delegando esa atención específicamente en quienes integran el Área de Agua, Tierra y Derechos Humanos, la cual se encarga de trabajar en relación con los conflictos que surgen en torno a la propiedad de la tierra (Marisa, comunicación personal, 12 de octubre de 2013).

En términos productivos, partiendo desde la mencionada escala local, la UST ha avanzado en la articulación de espacios a nivel provincial, e incluso nacional, por medio de redes de comercio justo. Estas prácticas han sido llevadas a cabo ante el desafío constante por lograr su sostenibilidad en un contexto socio-económico que, en términos generales, plantea serias dificultades para este tipo de unidades productivas. Se trata 
aquí de una escala acción (Lopes de Souza, 2013), basada en decisiones políticas de la organización. Frente a este panorama, en primer lugar, nos preguntamos por la forma más adecuada para ingresar -analíticamente hablando- al estudio de esta experiencia productiva e identificar el espacio producido por esa actividad. De la mano de este interrogante, a medida que fuimos desarrollando la investigación, surgieron otras preguntas relacionadas a aspectos teórico-metodológicos, a saber: ¿cuál es el valor que encierra el estudio de estas experiencias concretas teniendo en cuenta que, situadas localmente, llevan a cabo prácticas que se distanciarían de una lógica económica global?

Con estas inquietudes, nos lanzamos al trabajo de pesquisa geográfica con el concepto de lugar como principal guía de ingreso al tema. Como se dijo, la primera dimensión del lugar es la localización. Así, a la hora de indagar sobre los orígenes de la UST y, específicamente, de su Área de Producción y Comercialización, indefectiblemente nos vimos obligados a realizar una contextualización socio-espacial en torno a los diversos aspectos que posibilitaron la emergencia de la organización. Allí, factores socioeconómicos mundiales, nacionales, provinciales y departamentales entraron en juego y permitieron explicar su surgimiento desde su localización en Lavalle, más específicamente en el distrito de Jocolí (Jurado, 2018). A nivel provincial, por ejemplo, se pueden mencionar una serie de problemas estructurales representados principalmente en "la escasez de recursos hídricos y de la dificultad del acceso a la tierra" (Liceaga, 2008:70). En el caso particular de Jocolí, más del 6o \% de la superficie con derecho de riego está improductiva. Sin embargo, las razones para el surgimiento de una organización campesina como la UST -y con ella el despliegue de un área productiva- no pueden restringirse únicamente a las condiciones socio-económicas locales. Desde ya que, a nivel nacional, no se pueden ignorar los efectos de la crisis socio-económica de 2001 tanto en el agravamiento de las condiciones de vida como en la puesta en marcha de alternativas productivas para resolver algunas necesidades básicas en la zona. Insistiendo en aquellos comienzos de la organización a principios de la década del 2000, recurrimos a las palabras de Marisa, quien pone en valor precisamente el aprovechamiento de los recursos de la zona:

[Se producía] tomate triturado, uva en almíbar, lo que se conseguía de materia prima, porque no la cosechaban, porque nos la regalaban. Entonces la uva... alguna tenía un parralito, ${ }^{5}$ entonces hacía uvas en almíbar. El tomate era alguien que no le terminaba de cosechar y lo íbamos a cosechar nosotros. El tomate había bastante en la zona, entonces lo que sobraba se industrializaba. Y después esto, había un árbol de damasco y se hacía mermelada de damasco (...) Entonces mermeladas y después habían unas compañeras, no sé, había una finca de peras por acá, entonces empezaron a hacer algunos productos como peras confitadas con zanahorias, unas cosas así que eran como muy creativas en relación con lo que se conseguía (M. Rodríguez, entrevista personal, 27 de noviembre de 2015).

Resulta interesante el testimonio de la referente, ya que apunta a la creatividad como insumo básico para la producción basada, fundamentalmente, en los recursos disponibles a nivel local.

5 El parral es un conjunto de tallos de parra que, sostenidos por un armazón, forman una cubierta. En la Región Cuyo se emplea como enredadera ubicada en patios o en zonas aledañas a la vivienda con el fin de obtener sombra y, a la vez, un fruto comestible como la uva. 
Asimismo, el actual modelo del agronegocio implementado en la Argentina, que responde a lógicas globales de producción, también brinda motivos para dicha emergencia organizativa. Este modelo se sustenta, principalmente, en la exportación de commodities, proceso profundizado en la década de 1990 (Boy, 2005; Teubal, Domínguez y Sabatino, 2005; Giarraca, 2003). Con eje en el cultivo de soja y otros granos a gran escala, este proceso tiene como una de sus consecuencias la expulsión de población de zonas campesinas, dada la baja proporción de mano de obra que requieren las labores culturales, debido al alto grado de tecnificación del proceso. Ante este panorama, la organización en el ámbito rural es una salida que tomó un número importante de campesinos y campesinas.

La segunda dimensión entiende al lugar - por su denominación en inglés- como un locale, es decir "una serie de localidades o escenas donde las actividades cotidianas se desarrollan" (Agnew, 2011:326, traducción del autor). En este sentido, la producción industrial de alimentos de la UST se lleva a cabo en dos fábricas con las que cuenta la organización en un lapso corto de tiempo -año a año- que va desde diciembre hasta marzo/abril. Una de estas fábricas -la más antigua- se ubica en Jocolí, al lado de la sede de la organización. Allí, se encuentra, además, una radio comunitaria y también un vivero, de donde se obtiene parte de la materia prima que se emplea para la producción de alimentos. Es decir, tanto la fábrica en sí misma, como todo el predio, se han convertido en una verdadera localidad (locale) entendida como un espacio de encuentro donde las acciones productivas, educativas, comunicacionales y, de manera general, políticas se han desenvuelto a la par de la vida de la organización dándole forma al mismo tiempo (Jurado, 2018:207).

Justamente con relación a la fábrica de Jocolí, una de las trabajadoras comenta:

Para mí es una experiencia muy linda estar trabajando acá porque... es lo que yo les decía... no hay patrones, acá podemos llegar y somos dueñas de entrar y salir cuando nosotras queremos (...) Esas son cosas que nos ayudan y nos sirven. O decimos "nosotras hoy nos juntamos y no vamos a trabajar (en la producción específicamente); nos vamos a poner a limpiar o vamos a hacer...” Y bueno, y esas cosas que nosotras vemos que no hace falta que nadie nos diga ni que tengamos un patrón que nos diga "hoy tiene que hacer esto". Lo hacemos porque a nosotras nos gusta y porque queremos. Esa es la diferencia (Instituto Nacional de Tecnología Industrial [INTI], 2011).

Esa cotidianidad, como una actividad agradable a la que refiere la trabajadora de la fábrica, ha colaborado con la generación de un definido sentido de lugar, la tercera dimensión interviniente en la constitución de los lugares. Se trata de la asociación del lugar como una única comunidad, paisaje y orden moral (Agnew, 2011:326, traducción del autor). Asimismo, el sentido de pertenencia se refleja en la forma de mencionar al espacio de la fábrica -y el trabajo que allí se realiza-: decir "Jocolí" o "la sede" basta para entender de qué se trata o a qué ámbito se hace alusión (Jurado, 2018).

De esta manera, considerando la noción de lugar como punto de partida, pueden desplegarse concomitantemente una serie de conceptos que permitan comprender un fenómeno que, aunque pequeño y localmente analizado, encierra cierta complejidad. 


\section{Consideraciones finales}

El estallido geográfico acaecido en el marco del giro espacial en las ciencias sociales precisa una re-lectura epistemológica y metodológica. La pregunta por la elección de los conceptos geográficos más adecuados para nuestras investigaciones y el dilema que eso significa ha sido la forma que hemos elegido para contribuir a esa revisión teórica. En particular, hemos optado por poner en debate los alcances de los conceptos de territorio y lugar. ¿Qué factores inciden para que elijamos uno u otro en nuestros análisis?

De manera general, podemos afirmar que existen tres elementos que influyen en esta elección: la dimensión ontológica del sujeto-objeto de investigación, nuestras inquietudes teórico-metodológicas reflejadas en la pregunta-problema de investigación, y los aspectos políticos intervinientes en nuestro trabajo. Sin embargo, el recorrido de este texto y, en particular, el desarrollo breve de los estudios de caso, nos demuestran el gran peso de los dos últimos aspectos a la hora de definir los conceptos a elegir. Por un lado, en el plano político, como afirma Liceaga para el caso de la disputa de tierras en Los Leones, "pudo subrayarse la inherente politicidad que impregna la construcción de la territorialidad campesina, acentuada y puesta de relieve en situaciones de conflicto" (2017:241). Territorio puede asociarse, en definitiva, al conflicto y las disputas espaciales bajo relaciones de poder. En cambio, en el caso de las experiencias productivas propiamente dichas, su estrecha relación con fenómenos locales permite ensayar conexiones con lo comunitario y lo identitario del lugar, sin que ello implique necesariamente un parroquialismo geográfico. En este sentido, la renovación de la mano de Massey (2004) permite vincular al lugar de las resistencias al capital, con los flujos globales pensándolos en una relación de afectación mutua, aunque bajo determinadas relaciones de poder. A pesar de estas diferencias, es menester reconocer que el establecimiento de un límite claro entre los conceptos de territorio y lugar no resulta tarea sencilla; tal vez puedan pensarse en un área de frontera donde ambas nociones se influyen y se afectan.

Más allá de estas consideraciones, nos animamos a afirmar que, en última instancia, lo que va a definir la elección de los conceptos será nuestra mirada como investigadores/ as (que también está influenciada por consideraciones políticas), materializada en la pregunta de investigación. Como señala Raffestin, "hubiéramos querido más libros que preguntaran que libros que respondieran. Es mediante el interrogatorio, y no por las respuestas, que la medida del conocimiento se logra" (Raffestin, 1993:8, en Haesbaert, 2014:28, traducción del autor). 


\section{Q Bibliografía}

» Agnew, J. (1987). Una alternativa teórica acerca del lugar y la política. En J. Agnew, Place and politics: the geographical mediation of state and society (pp. 2543). Londres: Routledge.

» Agnew, J. (2011). Space and Place. En J. Agnew y D. Livingstone (Eds.), The Sage Handbook of Geographical Knowledge (pp. 316-330). London: SAGE.

》 Altschuler, B. y Pastore, R. (2015). La economía social y solidaria, y los debates del desarrollo en clave territorial. Reflexiones sobre experiencias y desafíos a partir de una práctica socioeducativa universitaria. Revista Idelcoop, Instituto de la Cooperación. Fundación de Educación, Investigación y Asistencia TécnicaIDELCOOP, 217, 11-29.

"Boy, A. (2005). Cambios productivos y sus repercusiones en el nivel agronómico. En N. Giarracay M. Teubal (Coord.), El campo argentino en la encrucijada (pp. 79100). Buenos Aires: Alianza.

》 Cresswell, T. (2011). Place-Part I. En J. Agnew y J. Duncan (Eds.), The WileyBlackwell Companion to Human Geography (pp. 235-244). ReinoUnido: John Wiley \& Sons.

» Dussel, E. (2005). Transmodernidad e interculturalidad. Interpretación desde la Filosofía de la Liberación. México: UAM.

»Elden, S. (2013). How Should We Do the History of Territory? Territory, Politics, Governance, 1(1), 5-20.

»Etxezarreta, M. (2014). Reflexionando sobre las alternativas. \#OtraEconomíaEstáEnMarcha. Economistas sin fronteras, 13, 11-15.

» Fernandes, B.M. (2004). Cuestión Agraria: conflictualidad y desarrollo territorial. Recuperado de: https://web.ua.es/en/giecryal/documentos/documentos839/ docs/bmfunesp-2.pdf

» Fernandes, B.M. (2005). Movimentos socioterritoriais e movimentos socioespaciais. OSAL, 6(16), 273-283.

» Fernandes, B.M. (2009) Sobre a Tipologia de Territórios. En M.A. Saquet y E.S. Sposito (Org.), Territórios e Territorialidades. Teoria, processos e conflitos (pp. 197-215). São Paulo: Expressão Popular. Traducido por María FranciAlvarez, Universidad Nacional de Córdoba.

» Giarraca, N. (10 de mayo de 2003). Radiografía del capitalismo agrario. Le Monde Diplomatique. Recuperado de: https://www.eldiplo.org/047-un-nuevo-pais-politico/ radiografia-del-capitalismo-agrario/

» González, H. (15 de mayo de 2016). El drama de las izquierdas. Página 12. Recuperado de: https://www.pagina12.com.ar/diario/ elpais/1-299397-2016-05-15.html

》Gould, P. (1987). Pensamiento sobre la geografía. Geocrítica. Cuadernos críticos de Geografía Humana, 12(68). Universidad de Barcelona. Recuperado de: http:// www.ub.edu/geocrit/geo68.htm. 
» Haesbaert, R. (2004). O mito da desterritorialização: Do "fim dos territorios"àmultit erritorialidade. Rio de Janeiro: Bertrand Brasil.

» Haesbaert, R. (2007). Território e Multiterritorialidade: um debate. Revista GEOgraphia, 17, 19-46.

》Haesbaert, R. (2009). 0 espaço como categoria e sua constelação de conceitos: uma abordagem didática (inédito). X Encontro Nacional de Prática de Ensino em Geografia, Porto Alegre.

》 Haesbaert, R. (2013). Del mito de la desterritorialización a la multiterritorialidad. Cultura y representacionessociales, 8(15), 9-42.

» Haesbaert, R. (2014). Viver no limite: território e multi/transterritorialidade em tempos de in-segurança e contenção. Rio de Janeiro: Bertrand Brasil.

» Halvorsen, S., Fernandes, B.M. y Torres, F. (2019). Mobilizing Territory: Socioterritorial Movements in Comparative Perspective. Annals of the American Association of Geographers, 109(5), 1454-1470.

》 Harvey, D. (2015). 0 espaço como palavra-chave. Pauta, Rio de Janeiro, 13(35), 126-52.

》 Hinkelammert, F. y Mora Jiménez, H. (2005). Hacia una economía para la vida (1 ${ }^{\text {a }}$ Ed.). Colección Economía Teológica. DEl: San José, Costa Rica.

» Hoelscher, S. (2011). Place-Part II. En J. Agnewy J. Duncan (Eds.), The WileyBlackwell Companion to Human Geography (pp. 245-259). ReinoUnido: John Wiley \& Sons.

» Instituto Nacional de Tecnología Industrial [INTI] (2011). Cadena de Valor del Tomate (Video). Sector Audiovisual, INTI - Comunicación y Participación Social. Recuperado de: https://www.youtube.com/watch?v=8HAt2sKZkb4\&t=18s

» Jurado, E. y Cardozo, L. (2016). La cuestión espacial en la Economía Social y Solidaria: una lectura de procesos cooperativos a partir de estudios geográficos. SaberEs, 8(1), 23-42

》 Jurado, E. (2018). Aspectos socio-espaciales de experiencias económicas alternativas: la economía social y solidaria en Mendoza de 2001 a 2017: un estudio desde los casos de El Arca y la Unión de Trabajadores Rurales sin Tierra. Tesis de doctorado. Universidad Nacional de Quilmes, Argentina.

»Lefebvre, H. (2013). La producción del espacio (1ª Ed.). España: Capitán Swing.

» Liceaga, G. (2008). "Tierra, Agua y Justicia”. Un análisis de la acción colectiva de la Unión de Trabajadores Rurales sin Tierra (Tesina de licenciatura sin publicar). Facultad de Ciencias Políticas y Sociales, Universidad Nacional de Cuyo, Mendoza, Argentina.

" Liceaga, G. (2017). Territorialidad y resistencias campesinas. El conflicto de "Los Leones" (Mendoza, Argentina). Tesis de doctorado. Centro de Estudios Avanzados, Universidad Nacional de Córdoba, Argentina.

»Lopes de Souza, M. (2012). 0 território: sobre espaço e poder, autonomia e desenvolvimento. En I.E. de Castro, P.C. da Costa Gomes y R.L. Corrêa, Geografia: conceitos e temas (pp. 77-116). Rio de Janeiro: Bertrand Brasil (15a. ed.).

"Lopes de Souza, M. (2013). Os conceitos fundamentais da pesquisa sócio-espacial

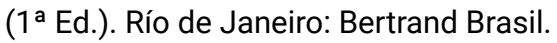


» Martínez, E. (2014). Configuración urbana, habitar y apropiación del espacio. Coloquio Internacional de Geocrítica, 13, Anales Barcelona: Universidad de Barcelona. Recuperado de: http://www.ub.edu/geocrit/coloquio2014/Emilio\%20 Martinez.pdf

" Massey, D. (2004). Lugar, identidad y geografías de la responsabilidad en un mundo en proceso de globalización. Treballs de la Societat Catalana de Geografia, $57,77-84$.

» Massey, D. (2008). Conferencia en Cendes. Geometrías internacionales del poder y la política de una "ciudad global": pensamientos desde Londres. Cuadernos del CENDES, 25(68), 115-122.

"Oslender, U. (1999). Espacializando resistencia: perspectivas de "espacio" y "lugar" en las investigaciones de movimientos sociales. Cuadernos de geografía, 8, 1-35.

》Piazzini, C.E. (2006). El tiempo situado: las temporalidades después del giro espacial. En D. Herrera y C.E. Piazzini (Eds.), (Des)territorialidades y (No)lugares: procesos de configuración y transformación social del espacio (pp. 53-73). Medellín: La Carreta.

»Pinto de Godoy, T. M. (2010). 0 espaço e o tempo da economia solidária. Biblio $3 W$, Revista Bibliográfica de Geografía y Ciencias Sociales, 15(886). Recuperado de: http://www.ub.edu/geocrit/b3w-886.htm

"Porto Gonçalves, C.W. (2009). Territorialidades y lucha por el territorio en América Latina. Geografía de los movimientos sociales en América Latina. Caracas: Instituto Venezolano de Ciencia y Tecnología (IVIC).

»Raffestin, C. (1993). Por uma geografia do poder. Tradução de Maria Cecília França. SP: Ática.

» Rincón Gamba, L. (2012). Territorios y sujetos de la economía social y solidaria. OtraEconomía, 6(10), 24-36.

"Sack, R.D. (1986). Human Territoriality: Its Theory and History. Cambridge: Cambridge UniversityPress. Traducción interna de la cátedra "Introducción a la Geografía", Universidad Nacional de Córdoba (1996).

»Saquet, M. (2013). El Desarrollo en una Perspectiva Territorial Multidimensional. Revista Movimentos Sociais e Dinâmicas Espaciais, 1, 111-123.

» Saquet, M. (2014). Território, territorialidade e desenvolvimento. Recuperado de: https://www.macroprograma1.cnptia.embrapa.br/agroecologia/programade-formacao-textos-de-apoio/programa-de-formacao-palestras/territorioterritorialidade-e-desenvolvimento-marcos-aurelio-saquet/view

》Santos, M. (2006). A natureza do espaço. Técnica e tempo. Razão e emoção. São Paulo: Editora da Universidade de São Paulo

»Soja, E. (1997). El Tercer espacio. Ampliando el horizonte de la imaginación geográfica. Geographikós,7(8), 71-76.

»Soja, E. (2010). Tercer espacio: extendiendo el alcance de la imaginación geográfica. En N. Benach, A. Albet i Mas y E.W. Soja: La perspectiva postmoderna de un geógrafo radical (pp. 181-209). Barcelona: Icaria.

» Teubal, M., Domínguez, D. y Sabatino, P. (2005). Transformaciones agrarias en la Argentina. Agricultura industrial y sistema agroalimentario. En N. Giarracay M. 
Territorio y lugar: la espacialidad en debate...

EMANUEL ALBERTO JURAdO

Teubal (Coord.), El campo argentino en la encrucijada (pp. 37-78). Buenos Aires: Alianza.

» Torres, F. (2011). Territorio y lugar: Potencialidades para el análisis de la constitución de sujetos políticos. El caso de un movimiento de desocupados en Argentina. Geograficando, 7(7), 209-238.

»Zanca, R. (2007). Territorialización alternativa y economía solidaria. La cotidianidad ascendente como gestora de ordenación sustentable a través de la experiencia comunitaria de la Asociación Mutual El Colmenar. Otra Economía, $1(1), 28-45$.

»Zusman, P. (2002). Milton Santos. Su legado teórico y existencial (1926-2001). Doc. Anàl. Geogr.,40, 205-219.

\section{Emanuel Alberto Jurado / emanueljurado@hotmail.com}

Doctor en Ciencias Sociales y Humanas por la Universidad Nacional de Quilmes. Jefe de Trabajos Prácticos en la asignatura Geografía e integración territorial, Licenciatura en Logística, Facultad de Ciencias Económicas, UNCuyo. Integrante del Grupo de Estudios Geografías Emergentes con sede en el Instituto de Geografía, UBA. Su tema de investigación versa en torno a la Economía Social y Solidaria, sujetos y prácticas espaciales en Mendoza (Argentina). Sus principales publicaciones son: Propuestas analíticas para un estudio espacial de la economía popular y solidaria. Aportes desde la realidad Argentina (2016); y en co-autoría: Economía Social y Solidaria en Río Negro y Mendoza. Políticas públicas, sujetos y especialidades en debate (2017) y La cuestión espacial en la Economía Social y Solidaria: una lectura de procesos cooperativos a partir de estudios geográficos (2016). También ha ejerce como docente en el nivel terciario y ha brindado cursos de posgrado en la UNCuyo. 